\title{
O Uso de Multirrepresentação e Ciclos de Interação em uma Aula Virtual de Química
}

\section{The Use of Multirepresentation and Interaction Cycles in a Virtual Chemistry Class}

\section{Emerich M. Sousa e João C. M. Paiva}

Resumo: Objetivou-se neste trabalho a elaboração de aula virtual de química, referente ao tópico de gases reais, para o nível de ensino médio, potencialmente significativa para o aluno e que fizesse uso de ciclos de interação e multirrepresentação. A multirrepresentação foi usada para favorecer a abstração, complementar informação, refinar o entendimento, favorecer o uso com poder argumentativo e coordenar diferentes registros semióticos. Os ciclos de interação incluíram perguntas de iniciação, transições entre o mundo dos objetos e eventos e o mundo das teorias e modelos, relações entre distintos referentes, operações de discutir/explicar/ generalizar e diversas intenções do material. O estudo foi do tipo QUANT-qual, com design quasi-experimental. A amostra foi constituída por 153 alunos da segunda série do ensino médio de uma escola pública do Brasil. Utilizaram-se pré-teste e pós-teste idênticos, com questões abertas. Os alunos foram divididos em quatro grupos, e cada grupo interagiu com um tipo de aula virtual: sem inovações, contendo ciclos de interação, contendo multirrepresentação e os dois recursos simultaneamente. A informação levantada nos testes foi analisada de acordo com um guia validado. Os grupos que tiveram contato com as inovações apresentaram magnitude de efeito maior para a variável pontuação de produção de texto do que o grupo sem inovações. Na análise qualitativa, observou-se aumento do número de alunos que utilizaram adequadamente a multirrepresentação, com aumento da qualidade das representações. O estudo, apesar das suas limitações, deixa um fundamento animador quanto às inovações didáticas empreendidas. O entendimento de algumas relações na construção do discurso no ensino de química poderá contribuir, em futuras investigações, para a ampliação da investigação a outros conteúdos de química, outras ciências, outros níveis de escolaridade e ensino não formal.

Palavras-chave: Ensino-Aprendizagem de Química. Estudo dos Gases. Aprendizagem Significativa. Semiótica. Ciclos de Interação. Multirrepresentação.

\begin{abstract}
The aim was to develop virtual lesson for chemistry, referring to the topic of real gases at the secondary level, potentially meaningful for the student and to make use of interaction cycles and multirepresentation. Multirepresentation was used to favor abstraction, complement information, refine understanding, favor use with argumentative power, and coordinate different semiotic records. Interaction cycles included initiation questions, transitions between the world of objects and events and the world of theories and models, relationships between different referents, discuss/explain/generalize operations and diversifications of material intentions. The study was of type QUANT-qual and with quasi-experimental design. The sample consisted of 153 students who attended the second grade of high school in a public school in Brazil. They used identical pre-test and posttest, with open questions. Students were divided into four groups and each group interacted with a type of virtual lesson: without innovations, with interaction cycles, with multirepresentation, and with both resources. The information collected in the tests was analysed according to a validated guide. The groups that had contact with the innovations had a greater magnitude of effect for the variable of text production score than the group without innovations. In the qualitative analysis, we observed an increase in the number of students who used the multirepresentation adequately, with an increase in the quality of the representations. The study, despite its limitations, leaves an encouraging foundation for the undertaken didactic innovations. The understanding of some relations in the construction of discourse in chemistry teaching may contribute, in future studies, to the expansion of the investigation to other contents of chemistry, other sciences, other levels of education and non-formal education.
\end{abstract}

Keywords: Teaching and Learning of Chemistry. Gas Study. Meaningful Learning. Semiotics. Interaction Cycles. Multirepresentation

Emerich Michel de Sousa (emerich.sousa@ufv.br) é professor de Química no Colégio de Aplicação da Universidade Federal de Viçosa (CAp-Coluni) e doutor em Ensino e Divulgação das Ciências da Universidade do Porto (UP), Portugal. Viçosa, MG - BR. João Carlos de Matos Paiva (jcpaiva@ fc.up.pt), professor associado no Departamento de Química e Bioquímica, membro da Unidade de Ensino das Ciências da Faculdade de Ciências, coordenador do núcleo de "Cultura Científica, Multimédia e Educação" do Centro de Investigação em Química da UP (CIQUP), é diretor do Doutoramento em Ensino e Divulgação das Ciências da UP. Porto - PT.

Recebido em 03/11/2017, aceito em 31/08/2018

A seção "Cadernos de Pesquisa" é um espaço dedicado exclusivamente para artigos inéditos (empíricos, de revisão ou teóricos) que apresentem profundidade teórico-metodológica, gerem conhecimentos novos para a área e contribuições para o avanço da pesquisa em Ensino de Química. 
O potencial oferecido pelas tecnologias da informação e da comunicação (TICs) para melhorar o ensino das ciências tem sido bem documentado. O desafio central para os professores é integrar o uso das TICs ao processo pedagógico e utilizar a tecnologia com uma postura inteligente e crítica (Steiner e Mendelovitch, 2017). Cabe ao docente considerar uma perspectiva questionadora, criativa e dinâmica em sua proposta metodológica, de forma que as tecnologias não sejam inseridas como meros adereços às aulas, mas que integrem e contribuam para o processo educacional (Moreno e Heidelmann, 2017).

Ekici e Pekmezci (2015) defendem que o uso de experiências visuais, auditivas e táteis enriquece a educação científica, e as instruções devem incluir abordagens multimodais para atingir mais sentidos e favorecer uma aprendizagem mais permanente e eficaz. Para Bidarra e Rusman (2017), a vantagem é a flexibilidade da abordagem de ensino e um design instrucional que pode ser alterado pelo aluno de acordo com suas necessidades pessoais e o contexto de aprendizagem. Fozdar (2015) destaca as oportunidades para a partilha de informações, recursos e experiências, bem como o oferecimento de oportunidades de trabalho em rede com colegas, estudantes, tutores e a instituição. Para atingir esses objetivos, Hinostroza et al. (2016) reforçam a necessidade de desenvolver habilidades digitais de professores e alunos, especificamente aquelas associadas à busca e seleção de informações disponíveis na internet e ao desenvolvimento e apresentação de produtos de informação.

Problemas relacionados à implantação de tecnologias, à pesquisa e à adoção por parte dos professores e alunos têm sido relatados.

Almeida (2008), em um resgate da história das TICs na educação do Brasil e de Portugal, salienta que as práticas avançaram e superaram obstáculos, mas, apesar da crescente quantidade de equipamentos colocados nas escolas no Brasil e em Portugal, a concretização das ações mostra-se aquém dos objetivos e desejos. Os computadores continuam subutilizados por distintos motivos, que dependem menos da presença da tecnologia na escola e mais de aspectos político-pedagógicos e de uma adequada formação dos educadores.

Bidarra e Rusman (2017) destacam a grande oferta de tecnologias educacionais à disposição dos alunos: telefones celulares inteligentes, software de rede, aplicações de aprendizagem, recursos educacionais abertos, ferramentas colaborativas, ambientes imersivos, ferramentas de produção e distribuição de mídia. No entanto, mesmo que a maioria da geração de aprendizes de hoje use dispositivos digitais, aplicativos de internet e redes sociais em uma base diária, principalmente para comunicação e entretenimento, há pouco conhecimento de como usar essas ferramentas e mídia para tornar a educação científica mais significativa, eficaz e atraente. Além disso, como ressaltam Paiva e Costa (2010), os alunos geralmente estão extremamente interessados em recursos digitais, porém, muitas vezes, passam pelas aplicações de forma precipitada e apressada.
Giordan (2015), em levantamento dos artigos publicados na revista brasileira Química Nova na Escola (QNEsc), descobriu que, nos dez anos anteriores, foram publicados apenas sete artigos na seção Educação em Química e Multimídia, o que representa cerca de um artigo a cada cinco edições do periódico, o que está abaixo da média das demais seções. A popularização das tecnologias digitais não se traduziu em investigações no âmbito da educação química, o que pode ser indicativo de sua pequena repercussão nas salas de aula dessa disciplina.

Para Groff e Mouza (2008), criar ambientes de aprendizagem eficazes com tecnologia continua a ser um desafio para os professores. Kurt (2013) questiona por que razão os professores adotam práticas que utilizam tecnologia em um nível tão limitado. Cita algumas barreiras que impedem o uso da tecnologia disponível de forma eficaz em suas instruções: alguns professores acreditam que são incapazes de integrar a tecnologia em suas aulas devido à formação inadequada; alguns deles acreditam que não podem usar a tecnologia de forma eficiente porque não têm tempo suficiente; e alguns não podem usar a tecnologia porque as tecnologias disponíveis estão desatualizadas e, às vezes, acreditam não ter apoio suficiente. No tocante à formação dos professores, Aslan e Zhu (2017) e du Plessis (2016) chamam a atenção para a importância da capacitação dos futuros professores em integrar as TICs nas práticas de ensino.

Zhao et al. (2002) destacam algumas barreiras que dificultam a adoção de tecnologia em sala de aula. Groff e Mouza (2008) ampliaram a proposta de Zhao et al. (2002) e desenvolveram uma estrutura - o Inventário Individualizado para Integrar Inovações Instrucionais (i5) - que pode capacitar os professores para buscar soluções no início do processo e aumentar a probabilidade de ter sucesso com a integração tecnológica (Tabela 1).

Vários investigadores apontam algumas medidas no sentido de ultrapassar essas limitações. Para integrar tecnologia no ensino, Zhao et al. (2002) salientam alguns pontos importantes para os professores, como conhecer os recursos e as restrições de várias técnicas e o modo como as técnicas específicas podem apoiar suas próprias práticas de ensino e metas curriculares; saber como usar as tecnologias; a necessidade de perceber que a integração de tecnologia requer suporte de outros; e estar cientes das condições de habilitação da tecnologia que eles planejam usar - que fatores do contexto a fazem funcionar.

Koehler e Mishra (2009) alertam que o ensino efetivo com a tecnologia requer compreensão da representação dos conceitos que utilizam tecnologias, das técnicas pedagógicas que usam tecnologias de forma construtiva para ensinar o conteúdo, de como a tecnologia pode ajudar a corrigir alguns dos problemas que os alunos enfrentam e ser usada para desenvolver novas epistemologias ou fortalecer as antigas. 
Tabela 1: Fatores que influenciam o sucesso das inovações tecnológicas nas salas de aula, adaptado de Groff e Mouza (2008)

\begin{tabular}{cccccc}
\hline Legislativo & Professor & Projeto & Escola & Estudante & Tecnologia \\
\hline Política & Proficiência & Distância da prática & Infraestrutura de & Nível de conforto & Tráfego de dados \\
Legislação & tecnológica & do professor & recursos físicos & com a tecnologia & Conectividade \\
Pesquisa & Compatibilidade & Distância da prática & Infraestrutura de & Distância com & Incompatibilidade de \\
& tecnologia-pedagogia & da escola & recursos humanos & experiências \\
& Percepção da & Distância dos & Cultura local & anteriores \\
& dinâmica escolar & recursos existentes & & Crenças, atitudes e \\
& & Dependência de & & engamento \\
& pessoas & & \\
& Dependência de & & \\
& recursos & & \\
& & &
\end{tabular}

\section{Ciclos de Interação}

O processo de aprendizagem, de acordo com Mortimer e Scott (2002), não deve ser visto como a substituição das velhas concepções existentes antes do processo de ensino pelos novos conceitos científicos, mas como a negociação de novos significados num espaço comunicativo. Nesse contexto, deve existir uma preocupação em se utilizar um material que permita uma interação com alunos, para promover a construção do significado.

É importante que o uso das TICs incorpore os avanços no estudo da construção do discurso em sala de aula. Os recursos a serem considerados devem levar em consideração alguns dos compromissos que o professor tem na sua sequência de aula (Scott et al., 2011; Mortimer et al., 2005); a preocupação em promover a reflexão, no estudo individual, ou a discussão, quando no estudo em grupo, conforme citado por Paiva e Costa (2010); a garantia de que o conteúdo do discurso da sala de aula sofra modificações progressivas (Mortimer e Scott, 2002); a transição entre diferentes modelagens e níveis referenciais (Silva e Mortimer, 2010) e entre diferentes abordagens comunicativas, a fim de abordar diferentes objetivos de ensino (Aguiar et al., 2010; Mortimer e Scott, 2002).

A incorporação de perguntas no material tem a intenção de promover a reflexão, no estudo individual, ou a discussão, quando no estudo em grupo, conforme citado por Paiva e Costa (2010). As perguntas de iniciação (de produto, de escolha, de processo e de metaprocesso) (Mortimer et al., 2005) constituem-se em uma tentativa de conexão entre o assunto estudado e situações do mundo real reconhecidas pelo aluno e também em um incentivo ao engajamento emocional, com o estabelecimento de empatia com o material. Estimula a reflexão do aprendiz, que poderá buscar exemplos de sua própria experiência e com condições para relacionar novas informações, de maneira não arbitrária e não literal, com a própria estrutura cognitiva e permitindo que haja mais de uma voz a ser considerada na construção de sentidos.

As operações de descrição, explicação e generalização, de acordo com Silva e Mortimer (2010), ocorrem dentro de um determinado movimento pelo qual o conhecimento é trabalhado ao longo das interações até adquirir um acabamento final e constituir um enunciado, e essas discussões podem envolver aspetos observáveis e mensuráveis de um determinado sistema em análise, considerado o mundo dos objetos e eventos, ou podem fazer referência a entidades criadas por meio do discurso teórico das ciências, considerado o mundo das teorias e dos modelos. Concebe-se, ainda, que isso pode ser feito em três níveis referenciais: (i) referente específico, que corresponde a um objeto ou fenômeno em particular; (ii) classe de referentes, que corresponde a um conjunto de fenômenos ou objetos que apresentam características em comum; e (iii) referentes abstratos, que correspondem a princípios ou conceitos mais gerais que possibilitam pensar sobre fenômenos em particular ou classe de fenômenos.

Dentro do processo de interação do professor com os alunos, destacam-se as possibilidades de o professor introduzir e desenvolver a história científica e de o aluno ter condições de desenvolver sua visão do assunto e manifestar seu entendimento e, sempre que possível, rever o progresso da história científica, garantindo-se que o conteúdo do discurso da sala de aula sofra modificações progressivas. Na aula virtual, essa sequência pode ser implementada pela discussão de ideias relevantes do comportamento geral (classe de referentes), pelo trabalho com valores numéricos na equação reformulada com exemplos específicos (referentes específicos), para dar forma, selecionar e marcar ideias-chave, e, no final, pelo confronto dos resultados obtidos com as explicações já apresentadas anteriormente aos alunos, de modo a reforçar o enlace entre a abordagem fenomenológica e conceitual e revendo o progresso na história científica.

Cada estudante precisa ter a oportunidade de trabalhar as novas ideias, "especificando um conjunto de suas próprias palavras" em resposta a essas ideias, para que possa apropriar-se delas, tornando-as as suas próprias ideias (Mortimer e Scott, 2002). As transições entre interações dialógicas e de autoridade são fundamentais para apoiar a aprendizagem significativa do conhecimento disciplinar, pois diferentes objetivos de ensino são abordados (Aguiar et al., 2010).

Paiva e Costa (2010) ressaltam a importância de oferecer sugestões para ações e reflexões. Nesse sentido, Mortimer et al. 
(2005) destacam as perguntas de iniciação de processo - que incentivam uma formulação mais completa pelo aluno e podem gerar uma cadeia de interações.

Scott et al. (2011) ressaltam que entender uma ideia envolve colocar em contato ideias novas e outras já existentes, fazer conexões entre estas ideias e, a partir daí, o número e qualidade das conexões criadas determinam a profundidade e a extensão da compreensão. Isso tem ressonância com a aprendizagem significativa de Ausubel e contrasta com o processamento superficial e a aprendizagem mecânica, caracterizada pela memorização de fatos discretos e pela falta de integração ou vinculação com ideias existentes. Esses autores identificam três objetivos principais para essas conexões: apoiar a construção do conhecimento, promover a continuidade e incentivar o engajamento emocional.

\section{Multirrepresentação}

A fluência representacional envolve produzir significado usando combinações de modos de representação, incluindo representações verbais (baseadas em palavras), visuais (com base em diagramas e gráficos) e simbólicas (equações e fórmulas) (Hill e Sharma, 2015). O uso de multirrepresentações é fundamental para a resolução de problemas e o desenvolvimento da compreensão. Para ter sucesso dentro de uma disciplina, os alunos precisam de ser competentes com um formato de representação, escolher e usar representações individuais apropriadas e integrá-las quando necessário.

Edwards (2015) destaca que múltiplas representações valorizam a diferença ao proporcionar aos alunos diferentes oportunidades para desenvolver uma compreensão profunda dos conceitos. Quanto ao uso de simulações computacionais, López e Pintó (2017) salientam que, apesar de serem ferramentas educacionais efetivas com poder visual e comunicativo, a compreensão dos alunos sobre essas representações visuais representadas não pode ser dada como garantida, exigindo atenção por parte dos professores de ciência.

Ainsworth (1999) propõe três funções exercidas pelas multirrepresentações no processo de aprendizagem: (i) complementar e confirmar a compreensão de outra representação anterior; (ii) restringir e refinar a interpretação, limitando o foco do aluno àquilo que se deseja enfatizar; e (iii) promover abstração, viabilizar generalização e relacionar representações.

Duval (2012) expõe as três atividades cognitivas fundamentais ligadas à semiose: $(i)$ a formação de uma representação identificável como uma representação de um registro dado; (ii) o tratamento de uma representação, que é a transformação desta representação no mesmo registro onde ela foi formada é uma transformação interna a um registro; e (iii) a conversão de uma representação, que é a transformação dessa função em uma interpretação em outro registro, conservando a totalidade ou uma parte somente do conteúdo da representação inicial - é uma transformação externa ao registro de início.
Esse autor alerta para um isolamento de registros de representação, em todos os níveis de ensino, na grande maioria dos alunos. A ausência de coordenação entre dois ou mais registros não impede toda a compreensão, mas também não favorece em nada as transferências e as aprendizagens posteriores: torna os conhecimentos adquiridos pouco ou não utilizáveis em outras situações em que deveriam realmente ser empregados.

Para Laburú et al. (2011), uma aprendizagem significativa passa a prevalecer quando um mesmo conceito ou uma mesma proposição consegue ser expresso de diferentes maneiras, por meio de distintos signos ou de grupos de signos, equivalentes em termos de significados e que permitem a formação de vínculos entre os conhecimentos prévios do sujeito e os novos conceitos, possibilitando a estruturação de sentidos e de relações argumentativas.

Com base no uso de representações dos químicos, Kozma e Russell (2005) descreveram cinco níveis de competência representacional com base na geração, uso e compreensão das visualizações (Figura 1). Os cinco níveis variam desde o uso de características de superfície dos novatos para definir fenômenos químicos (nível 1) até o uso retórico dos especialistas em representações ou visualizações para argumentar, discutir e explicar fenômenos químicos (nível 5).

O desenvolvimento não é automático ou uniforme. Uma pessoa pode ser mais competente e mostrar habilidades de nível superior com um sistema específico (e.g. equações químicas) do que outra (e.g. gráficos). No entanto, ao longo do tempo e com orientação adequada, o aluno poderá avançar em suas habilidades, internalizá-las e integrá-las na prática regular. No exemplo a seguir são utilizadas representações correspondentes aos cinco níveis, a partir de um exemplo de compressão isotérmica dos gases (Figura 2).

$\mathrm{O}$ exemplo ilustra um recipiente com êmbolo contendo gás (Figura 2a), sobre o qual se aplica uma força externa (Figura 2b). A resultante externa sobre o êmbolo torna-se maior do que a força exercida pelas partículas gasosas (Figura 2c) presentes no recipiente. Se aumentarmos a compressão, o volume do recipiente diminui até determinado valor e é apenas ligeiramente maior que o volume total das moléculas (Figura 2d). Isso explica por que, em altas pressões, o gás real ocupa volume maior que um gás perfeito, pois na teoria cinética o volume das moléculas é desprezado (Figura 2e).

\section{O Problema}

A química, segundo Özmen (2004), tem sido considerada, por professores de química, pesquisadores e educadores, um assunto difícil para os jovens estudantes.

As questões fundamentais levantadas e que motivaram o estudo foram: (i) em sala de aula, o discurso científico que se instaura e se constrói é feito por um somatório de ações que envolvem as intenções do professor, as operações de descrição, explicação e generalização, a escolha de modelos apropriados e 


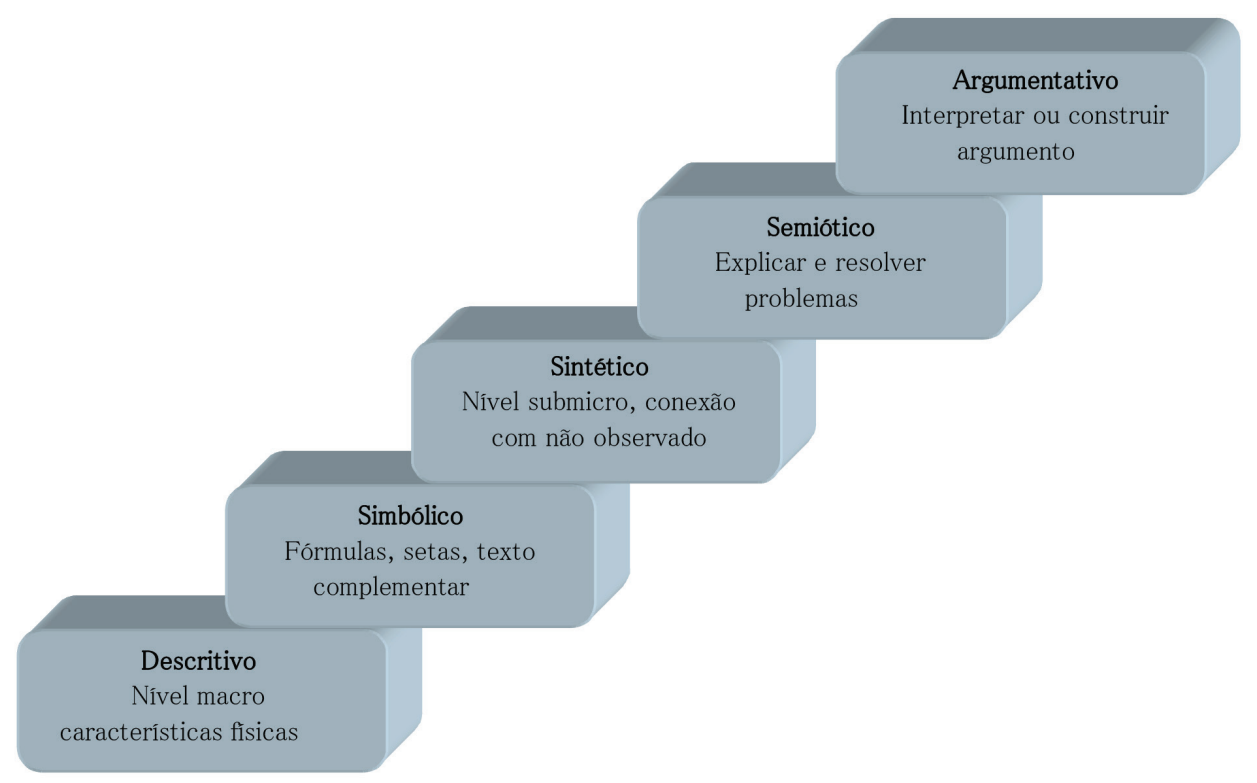

Figura 1: Níveis de competência representacional, segundo Kozma e Russell (2005).

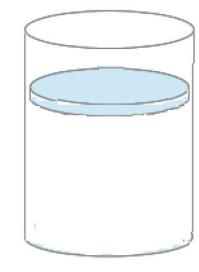

(a)

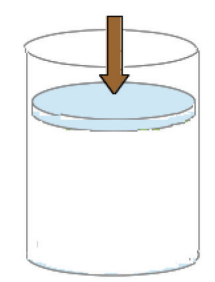

(b)

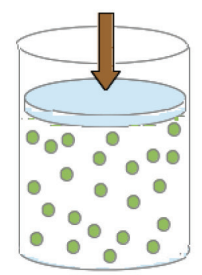

(c)

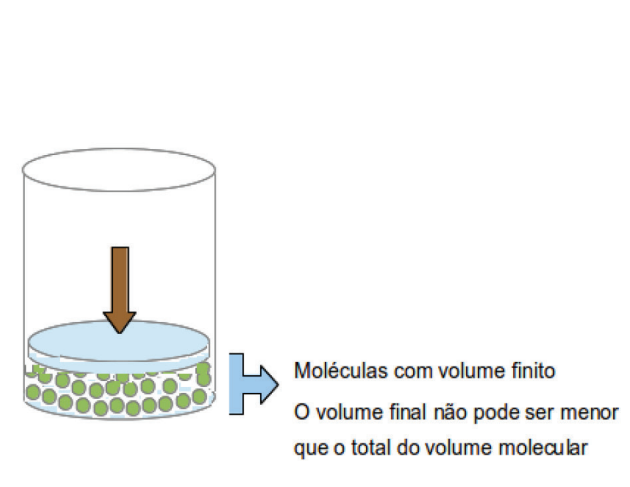

(d)

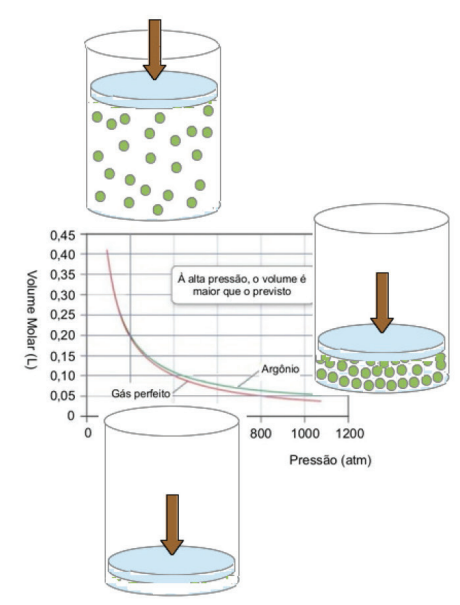

(e)

Figura 2: Ilustrações dos cinco níveis de competência representacional, a partir de Kozma e Russell (2005).

de níveis de referência, a abordagem comunicativa, os tipos de interações e as conexões entre as ideias - como transpor essa riqueza e complexidade para um material de aprendizagem?; (ii) espera-se que, durante a aprendizagem das ciências, o aluno desenvolva a sua fluência representacional e que possa não somente usar as representações para explicar relações entre propriedades e processos, mas também explicar a preferência por um tipo de representação em detrimento de outros - como conseguir que um material de aprendizagem tenha potencial para desenvolver essa competência representacional nos alunos?; e (iii) é desejável que o aluno consiga estabelecer significados novos a partir da interação entre seus conhecimentos prévios e o material de aprendizagem, e, para isso, o material deve fornecer condições para que a aprendizagem seja significativa, isto é, não arbitrária e não literal (Ausubel, 2003) - como utilizar esses recursos na criação de um material potencialmente significativo? 
Objetivou-se neste trabalho a criação de material específico para o uso em ambiente virtual de aprendizagem, aliando tecnologia e recursos discursivos, que pudesse agregar valores à formação dos alunos do colégio, estender o contato com o conteúdo de química para além do horário de aula e servir de referência para licenciandos em experiência de prática escolar. O material foi caracterizado como uma aula virtual, que se refere a período estruturado de tempo destinado a abordar um determinado assunto ou atividade e que está disponível em um ambiente virtual de aprendizagem.

\section{Metodologia}

O estudo apresentou uma componente de análise quantitativa, trabalhando com dados numéricos e submetidos à análise estatística e outra componente de análise qualitativa, observando o nível de competência representacional dos alunos. Assim, justifica-se classificar este estudo como QUANT-qual, uma abordagem mista, mas com preponderância quantitativa (MacCarthy et al., 2013; Hernández Sampieri et al., 2010).

Recorreu-se a um método de amostragem não probabilístico - amostragem de conveniência. A amostra foi constituída por 153 alunos, que se encontravam interessados no estudo e disponíveis para colaborar, de um total de 168 alunos que cursavam a segunda série do ensino médio. Os alunos desta série apresentavam média de idade de 16,4 anos, com 50,6\% do sexo feminino e $49,4 \%$ do sexo masculino. $O$ acesso ao colégio é muito concorrido, e isso pode ser notado na porcentagem de alunos que já haviam cursado a primeira série $(45,8 \%)$ ou mesmo a segunda $(1,8 \%)$ e optaram por reiniciar o ensino médio, repetindo a primeira série. Quase a totalidade dos participantes tem acesso à internet $(99,4 \%)$ e computador em casa $(97,6 \%)$. Quanto ao grau de instrução da mãe, $27,7 \%$ responderam ter até o ensino superior completo e $32,5 \% \mathrm{com}$ o ensino superior completo e pós-graduação (especialização, mestrado ou doutoramento). Em relação ao grau de instrução do pai, $19,9 \%$ responderam ter até o ensino superior completo e $16,9 \%$ com o ensino superior completo e pós-graduação. Apenas 13,2\% dos alunos são originários da própria cidade onde o colégio se situa, e os demais são provenientes de outras cidades.

Dentro da classificação dos designs experimentais, o presente trabalho classificou-se como quasi-experimental (Tuckman, 2012; Hernández Sampieri et al., 2010). Limitações práticas relativamente às oportunidades para selecionar ou designar os participantes e para manipular as condições impediram um controle experimental completo. O estudo decorreu em contexto escolar, com turmas já formadas, sem a possibilidade de constituir aleatoriamente os grupos necessários à investigação e, por questões éticas, ficou impossibilitado de administrar a intervenção a uma parte dos alunos, recusando-a à outra.

Utilizou-se como técnica de coleta de dados o inquérito por questionário com questões abertas, objetivando a comparação entre os grupos com base nas respostas dadas ao questionário usado como pré-teste e pós-teste. As questões abertas serviram a um duplo propósito: análise segundo critérios estabelecidos (McDermott e Hand, 2013), de modo a obter uma pontuação e posterior análise estatística, e avaliação qualitativa das respostas, permitindo classificar o nível de competência representacional (Kozma e Russell, 2005), a função exercida pela multirrepresentação (Ainsworth, 1999) e as atividades ligadas à semiose (Duval, 2012). Esta opção possibilitou obter mais informações nas respostas, com mais detalhes e sem limitar a capacidade de exposição do aluno. As perguntas abertas eram as mais adequadas para a análise de produção de texto, a utilização de múltiplos modos (verbal, visual e simbólico) e a forma de incorporação destes modos ao texto, que era o pretendido no trabalho. Desvantagens como tempo envolvido na interpretação das respostas e exigência de experiência do avaliador foram levadas em consideração, mas foram julgadas como contornáveis. A maior desvantagem seria a dificuldade de análise de uma maneira estatística; segundo Cohen et al. (2007), os dados não seriam facilmente comparados entre os participantes, e as respostas seriam difíceis de codificar e classificar. Essa dificuldade foi enfrentada a partir da escolha de um guia de correção adequado e validado, com critérios bem estabelecidos para analisar cada questão e de modo que a nota final representasse um somatório de vários itens analisados padronizadamente.

Os alunos assistiram às aulas normais do turno matutino, no dia da intervenção. Após um intervalo para o almoço, retornaram à tarde para a realização do pré-teste, exposição à aula virtual e realização do pós-teste, idêntico ao pré-teste.

As aulas virtuais foram elaboradas especificamente para a investigação, utilizando-se o KompoZer versão $0.8 \mathrm{~b} 3$, multiplataforma, disponível para Linux, MacOS e Windows, seguindo os critérios de usabilidade técnica (Nielsen, 2003) e pedagógica (Nokelainen, 2006). Elas foram acessadas a partir de computador, através do arquivo HTML correspondente em modo offline.

O material abordou o tema de gases reais por ser um assunto pouco explorado no ensino fundamental, permitindo a investigação de conhecimento preeexistente e a avaliação de melhorias nas respostas dos alunos. Foi concebido para uma aprendizagem significativa e por recepção (Novak e Gowin, 1996), e a aula foi dividida em seis telas: Apresentação, Gases reais, Desvios de comportamento, Fator de compressibilidade, Equação de van der Waals e Referências. O conteúdo de química foi elaborado a partir de Tro (2015), Chang e Goldsby (2011), Petrucci et al. (2011), Levine (2009), Mortimer (2008), Barker (2007), Atkins e de Paula (2006), Engel e Reid (2006), Brown et al. (2004), Alcañiz (2003) e Castellan (2001).

Foram criados quatro tipos de aula virtual: sem inovações (1), contendo ciclos de interação (2), contendo multirrepresentação (3) e os dois recursos simultaneamente (4). As Figuras 3, 4, 5 e 6 ilustram a tela Fator de compressibilidade para cada uma das aulas, enfatizando as principais diferenças entre elas. 
No estudo dos gases perfeitos, utilizamos bastante a equaçāo de estado, lembra-se dela?

Para um mol $(n=1)$ de gás perfeito, $P V=n R T$ torna-se $P V=R T$.

Uma forma de comparar um gás real com um gás perfeito hipotético é fazer uma série de medidas de $\mathrm{P}, \mathrm{V}$ e T em um mol de gás e entāo avaliar a quantidade PV/RT para cada medida.

Para o gás perfeito, a relaçāo PV/RT $=1$. Para um gás real, a relaçāo PV/RT fica diferente de 1 , dependendo do desvio de comportamento desse gás.

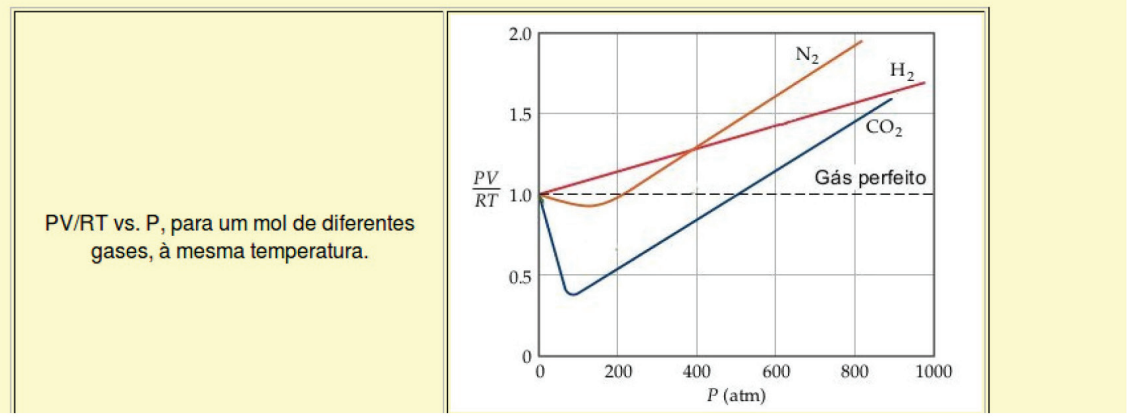

Figura 3: Tela Fator de compressibilidade para a aula virtual sem inovações.

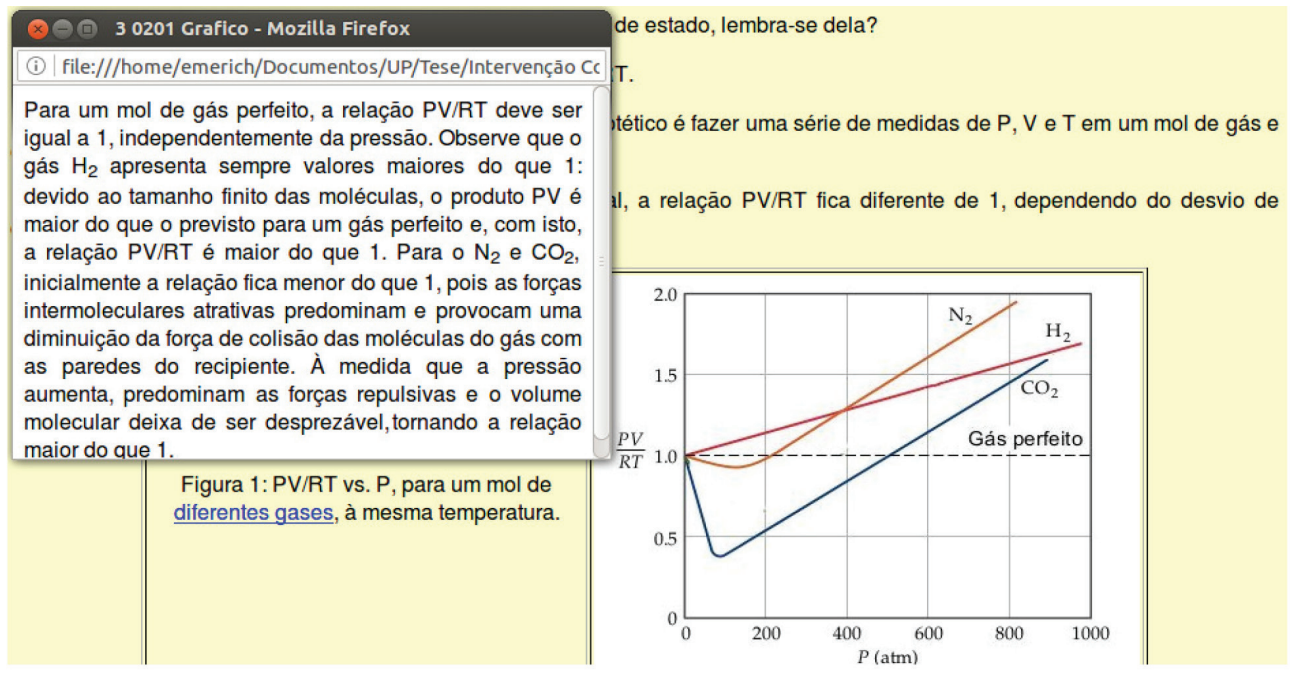

Figura 4: Tela Fator de compressibilidade para a aula virtual contendo ciclos de interações.

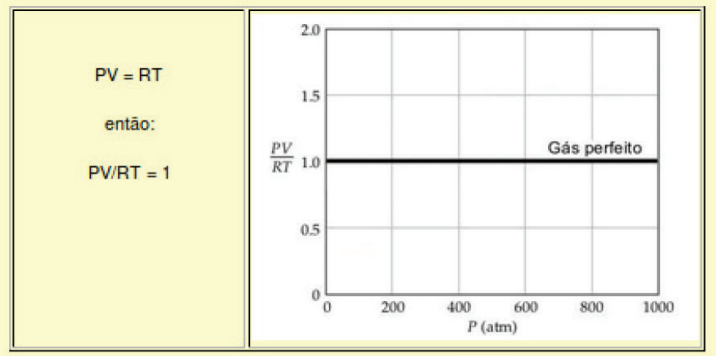

Para um gás real, a relação PV/RT fica diferente de 1, dependendo do desvio de comportamento desse gás. Observe os gráficos PV/RT vs. P, para um mol de diferentes gases, à mesma temperatura.

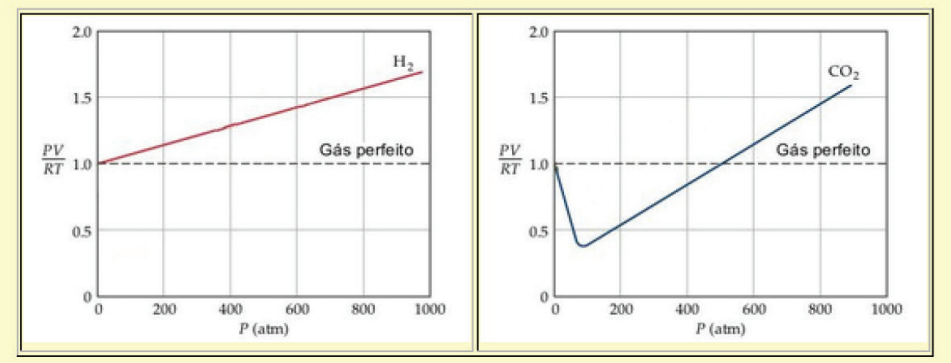

Figura 5: Tela Fator de compressibilidade para a aula virtual contendo multirrepresentação. 


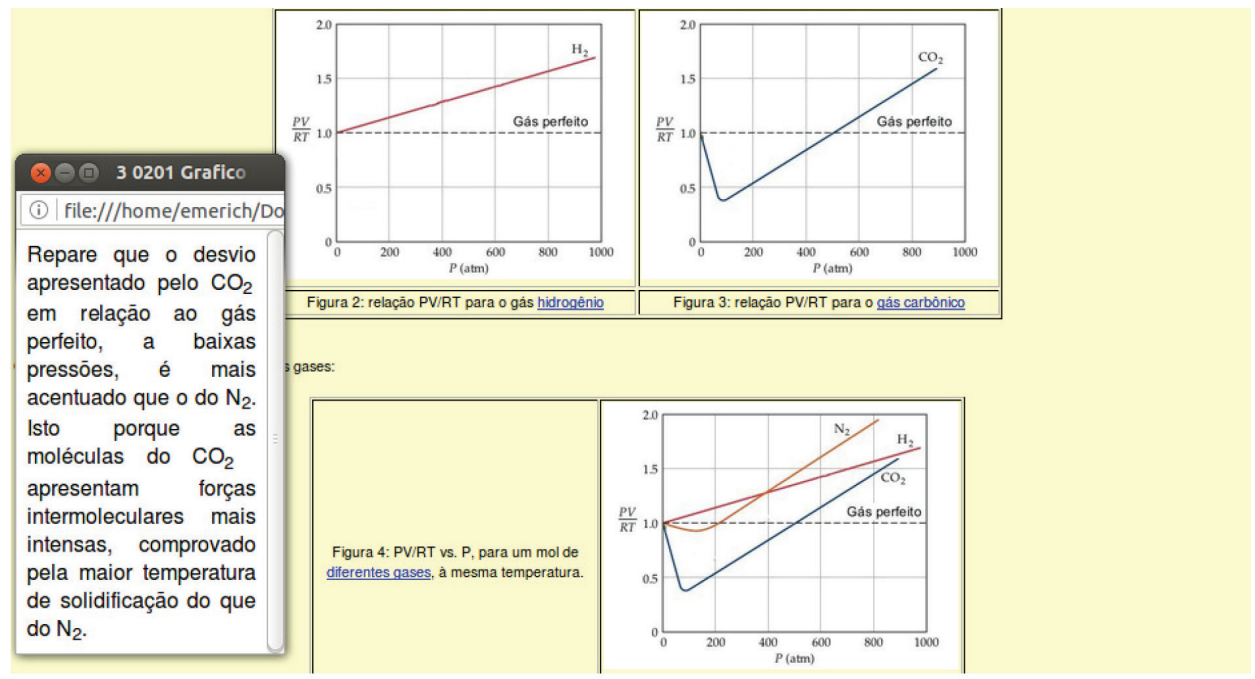

Figura 6: Tela Fator de compressibilidade para a aula virtual contendo multirrepresentação e ciclos de interações.

A tela inicia com uma questão, que é uma tentativa de resgatar a base conceitual dos alunos e favorecer o movimento no sentido da construção de novas concepções que serão desenvolvidas na sequência, corresponde, também, a um objetivo de promover a continuidade, conforme destacado por Scott $e t$ al. (2011), ao estabelecer um elo entre diferentes pontos no tempo. Apresenta-se a equação de estado $(\mathrm{PV}=\mathrm{nRT})$, atribuise valor $(n=1)$ e rearranja-se a equação $(P V / R T=1)$, o que caracteriza uma atividade de tratamento, em que a representação sofre transformações internas ao próprio registro. Segue-se uma generalização dos gases reais para, na sequência, em um movimento de recontextualização com referentes específicos, se fazer a transformação dessa representação em um outro registro, agora gráfico, o que caracteriza uma conversão, que é uma representação externa ao registro de início.

$\mathrm{O}$ recurso interativo proposto tem o objetivo de auxiliar o aluno no entendimento do gráfico, à semelhança do comportamento que um professor poderia assumir em sala de aula, ao mostrar o traçado de cada ente e estabelecer relações com a teoria discutida previamente. Busca o favorecimento de conexões entre as ideias, com o objetivo de apoiar a construção do conhecimento, à semelhança do que é discutido por Scott et al. (2011).

A opção de multirrepresentação foi a substituição de um gráfico típico, com várias substâncias representadas ao mesmo tempo, por vários gráficos, cada um com uma substância representada separadamente. A interpretação dos gráficos exige a conexão entre os domínios fenomenológico e conceitual, o que já se constitui em um grande desafio. O recurso representacional proposto tem o objetivo de auxiliar o aluno no entendimento do assunto, à semelhança do comportamento que um professor poderia assumir em sala de aula, ao mostrar o comportamento de cada substância separadamente e depois representar uma síntese do que foi apresentado. Ao mostrar a relação entre representações, a multirrepresentação cumpre o objetivo de permitir aprofundar o entendimento.
Os gráficos foram adicionados para permitir e reforçar a conexão com o mundo dos objetos e eventos e recebem esclarecimentos adicionais para permitir a transição entre classes de referentes para um referente específico. A caixa de texto utiliza referentes específicos, abordados no gráfico, para tornar mais evidente a diferença de comportamento entre eles, e prepara caminho para futuras generalizações, que ocorrerão em um momento pertinente do material.

Recorreu-se ao software de apoio Excel para digitação dos dados e cálculos preliminares e ao Statistical Package for the Social Sciences (SPSS), v. 24.0, para a análise estatística descritiva e inferencial. Field (2009) cita quatro premissas para os testes paramétricos: a distribuição deve ser normal, a variância deve ser homogênea, os dados devem ser medidos em nível de intervalo e o comportamento de um participante não deve influenciar o comportamento de outro. Na presente investigação, os dados seguiram distribuição não normal, justificando a aplicação de testes não paramétricos. A desvantagem destes testes é que não são tão potentes quanto os paramétricos, sendo possível que, mesmo havendo diferenças entre os dados, elas não tenham sido detectadas.

Utilizou-se o relatório de magnitude de efeitos, de acordo com recomendação da Associação Americana de Psicologia (APA) (Barry et al., 2016). A magnitude de efeito, de acordo com Durlak (2009), avalia a grandeza ou a força dos resultados que ocorrem nos estudos de pesquisa e fornece uma medida sem escala, que reflete a significância prática da diferença ou a relação entre as variáveis (Maher et al., 2013).

\section{Resultados Quantitativos}

Do conjunto estudado, destacam-se os resultados para três variáveis.

A variável Pontuação de Produção de Texto (PPT) consistiu no somatório de outras quatro variáveis (Obrigatoriedade, Correção gramatical, Exatidão e Completude), avaliadas para 
cada questão e com pontuação igual a 0 (ausência desta característica), 1 (característica pouco presente), 2 (característica frequente) e 3 (característica sempre presente). Os grupos que tiveram contato com as diferentes aulas virtuais não mostraram diferenças, na partida. Os resultados no pós-teste foram significativamente maiores do que no pré-teste. Os grupos, no entanto, não apresentaram diferença estatisticamente significativa entre si, no pós-teste.

A magnitude de efeito da variável Pontuação de Produção de Texto apresentou valores maiores para os grupos que tiveram contato com os materiais com inovações (ciclos de interação e multirrepresentação). Como a capacidade de qualquer teste de significância para detectar um efeito depende inteiramente da potência estatística proporcionada pelo tamanho da amostra (Maher et al., 2013), no presente estudo, em que as amostras eram pequenas, a análise da magnitude de efeito ofereceu confiança aliada à significância prática da diferença entre as variáveis.

A variável Índice de Incorporação (II) foi criada para fornecer uma medida quantitativa da qualidade científica da resposta e do grau de incorporação de multirrepresentações. Levou em conta a capacidade do estudante para utilizar o texto de forma adequada, para empregar vários modos de representação de forma eficaz e para utilizar estratégias no sentido de ligar outros modos não texto com o texto. Os grupos que tiveram contato com as diferentes aulas virtuais não mostraram diferenças, na partida. Os resultados no pós-teste foram significativamente maiores do que no pré-teste. O pequeno contato dos alunos com a aula virtual (uma sessão de $50 \mathrm{~min}$ ) pode não ter sido suficiente para sensibilizá-los e deixá-los fluentes quanto ao uso de representações não texto. Apesar disso, a variável Índice de Incorporação (II) apresentou resultado próximo do significativamente aceitável $(p=0,06)$, quando da comparação entre os diferentes grupos.

A variável identificada por Índice de Evolução Formativa (IEF) foi criada com o objetivo de analisar a performance global do estudante e incidiu sobre a essência do processo que transcorreu durante a intervenção, incluindo alguns medidores relevantes para o desempenho do aluno, importantes no dia a dia escolar, como aprovação, eventual influência da aula virtual na estruturação de respostas - com termos e conceitos transmitidos pelo material -, capacidade de representação em gráficos e melhoria na qualidade das respostas (mais completas e com maior exatidão científica). $\mathrm{O}$ sistema de pontuação das características analisadas derivou daquele utilizado na investigação (de 0 a 3), acrescido de sinal para contabilizar apoio ou prejuízo para o processo de ensino e aprendizagem. Considerou-se pontuação +3 se a qualidade medida estava presente e contribuiu para o processo de aprendizagem; zero, se a qualidade estava ausente; -3 , quando a qualidade medida estava presente, mas comprometeu o processo de aprendizagem. O somatório dos valores para o IEF poderia assumir: valor negativo, indicando prevalência de efeitos indesejáveis (como diminuição da taxa de aprovação, não ocorrência de acréscimos de exatidão e completude das respostas); valor zero, indicando que efeitos indesejáveis anularam os desejáveis; e valor igual ou maior que 3 , indicando que os efeitos desejáveis foram superiores. $\mathrm{Na}$ presente investigação, valores iguais ou maiores que 3 foram obtidos por $54 \%$ dos alunos apresentados à aula virtual 1 (aula sem inovações), 68\% dos alunos apresentados à aula virtual 2 (aula com multirrepresentação), 88\% dos alunos apresentados à aula virtual 3 (aula com ciclos de interação) e $79 \%$ dos alunos apresentados à aula virtual 4 (aula com ciclos de interação e multirrepresentação).

Constatou-se, através do teste de Kruskal-Wallis, que a variável IEF foi significativamente afetada pelo tipo de material. Testes post hoc (Mann-Whitney e o de soma de postos de Wilcoxon) indicaram que os IEFs dos alunos que interagiram com os materiais 3 (ciclos de interação) e 4 (ciclos + multirrepresentação) foram significativamente maiores do que o IEF dos alunos que interagiram com o material 1 (tradicional). Considerou-se, portanto, que a aula virtual contribuiu para uma evolução formativa dos alunos.

\section{Resultados Qualitativos}

A análise qualitativa das respostas mostrou-se mais útil para perceber alguma influência da aula virtual sobre a competência representacional. Observou-se aumento do número de alunos que utilizaram adequadamente a multirrepresentação, e é preciso ressaltar o aumento da qualidade das representações e o seu uso para funções de promover abstração, viabilizar generalização e relacionar as representações, muitas vezes com características argumentativas.

Ao todo, 18 alunos utilizaram a multirrepresentação no pré-teste. As representações não texto corresponderam a equação química (1 vez), fórmulas matemáticas (2 vezes) e figuras (15 vezes). As figuras situaram-se nos níveis icônico (1 vez), simbólico (11 vezes) e sintático ( 2 vezes), de acordo com a classificação de Kozma e Russell (2005). Desse total, apenas seis alunos usaram multirrepresentação apropriadamente.

No pós-teste, 12 alunos utilizaram a multirrepresentação, dos quais 11 fizeram-no de maneira apropriada. As representações não texto corresponderam ao uso de gráficos (2 vezes) e fórmulas matemáticas (2 vezes), todos no nível argumentativo, e figuras (8 vezes), com uso nos níveis simbólico e sintático.

Verificou-se, portanto, aumento do número de alunos que utilizaram adequadamente a multirrepresentação, além do aumento da qualidade das representações e do uso para funções de promover abstração, viabilizar generalização e relacionar as representações, o que, segundo Ainsworth (1999), permite um profundo entendimento da situação. Essas representações foram usadas de forma original, com critérios que os alunos julgaram importantes para sua escolha.

Observou-se, no pós-teste, a utilização de termos específicos empregados na aula virtual e de explicações construídas a partir 
Tabela 2: Uso apropriado de representações e de termos e explicações embasados na aula virtual, em número absoluto de ocorrência, em cada uma das aulas virtuais, no pós-teste

\begin{tabular}{lccc}
\hline Aula virtual & Representações não-texto & Termos & Explicações \\
\hline Sem inovações & 3 & 2 & 1 \\
Multirrepresentação & 3 & 1 & 2 \\
Ciclos de interação & 3 & 2 & 5 \\
Multi + ciclos & 3 & 1 & 7 \\
\hline
\end{tabular}

da lógica do material. As expressões foram usadas dentro das respostas elaboradas pelos alunos, sinalizando uma apropriação com contextualização e internalização de conceitos, sem ser simplesmente por memorização. A Tabela 2 apresenta o uso apropriado de representações e de termos e explicações fundamentados na aula virtual, no pós-teste.

Observa-se na Tabela 2, nos alunos que utilizaram a aula virtual com recurso de ciclos de interação apenas e no material com recurso de ciclos de interação e multirrepresentação, um aumento do uso de explicações construídas a partir de argumentos expostos no material.

\section{Considerações Finais}

Tuckman (2012) afirma que o tamanho da amostra deve ser capaz de evidenciar a diferença, caso exista, prevista na hipótese. O presente estudo teve à disposição um número limitado de alunos, e isso pode, segundo Maher et al. (2013), ter comprometido a eficiência dos testes estatísticos e diminuído a capacidade de significância para detectar um determinado efeito.

A experiência do pré-teste, de acordo com Tuckman (2012), pode aumentar a probabilidade de os sujeitos melhorarem o seu desempenho no pós-teste subsequente, principalmente no caso desta investigação, em que o pós-teste foi idêntico ao pré-teste e aplicado após curto intervalo de tempo. Assim, fica a dúvida sobre quão a melhoria no desempenho observado foi consequência da intervenção (aula virtual) ou do pré-teste. Além disso, ainda com base em Tuckman (2012), em outro conjunto de condições em que o pré-teste não tivesse sido aplicado, o tratamento poderia não ter os mesmos efeitos, o que afeta a generalização dos resultados.

O entendimento de algumas relações da construção do discurso no ensino de química, levantadas nesta investigação, poderá contribuir, em futuros estudos, para a ampliação de aplicabilidade em outras ciências e em outros níveis de escolaridade.

A utilização de equipe multidisciplinar, com a participação de professores, especialistas em tecnologia, profissionais da área de educação química e ensino de ciências, poderá possibilitar a otimização da implementação dos recursos propostos, aliados a outros que, porventura, sejam pertinentes.

$\mathrm{O}$ estudo tem grande potencial para ser utilizado com professores em formação, estabelecendo-se uma linha de investigação quanto à aceitação, perspectiva e possibilidades de uso de aula virtual e quanto às concepções dos futuros professores a respeito dos recursos de multirrepresentação e ciclos de interação.

Espera-se que a experiência advinda desta pesquisa em ensino de ciências possa contribuir para oportunos debates também no campo da divulgação de ciências, pois as discussões sobre multirrepresentação e ciclos de interação podem contribuir para o desenvolvimento de formas de suporte ao processo de construção de significados no ensino não formal.

\section{Referências}

AGUIAR, O. G.; MORTIMER, E. F. e SCOTT, P. Learning from and responding to students' questions: the authoritative and dialogic tension. Journal of Research in Science Teaching, v. 47, n. 2, p. 174-193, 2010.

AINSWORTH, S. The functions of multiple representations. Computers \& Education, v. 33, n. 2-3, p. 131-152, 1999.

ALCAÑIZ, E. J. Enlace químico y estructura de la materia. Universidad de Alcalá, 2003. Disponível em http://www3.uah.es/edejesus/ resumenes/EQEM.htm, acessada em Novembro 2018.

ALMEIDA, M. E. B Educação e tecnologias no Brasil e em Portugal em três momentos de sua história. Educação, Formação \& Tecnologias, v. 1, n. 1, p. 23-36, 2008.

ASLAN, A. e ZHU, C. Investigating variables predicting Turkish preservice teachers' integration of ICT into teaching practices. British Journal of Educational Technology, v. 48, n. 2, p. 552-570, 2017.

ATKINS, P. W. e DE PAULA, J. Atkins' physical chemistry. $8^{\mathrm{a}}$ ed. Oxford: Oxford University Press, 2006.

AUSUBEL, D. P. Aquisição e retenção de conhecimentos. Lisboa: Plátano Edições Técnicas, 2003.

BARKER, B. Peterson's master AP chemistry. $2^{\mathrm{a}}$ ed. Lawrenceville: Peterson's, 2007.

BARRY, A. E.; SZUCS, L. E.; REYES, J. V.; JI, Q.; WILSON, K. L. e THOMPSON, B. Failure to report effect sizes: the handling of quantitative results in published health education and behavior research. Health Education and Behavior, v. 43, n. 5, p. 518-527, 2016.

BIDARRA, J. e RUSMAN, E. Towards a pedagogical model for science education: bridging educational contexts through a blended learning approach. Open Learning, v. 32, n. 1, p. 6-20, 2017.

BROWN, T. L.; LEMAY JR., H. E.; BURSTEN, B. E. e BURDGE, J. R. Química. La ciencia central. Naucalpan de Juárez: Pearson Educación, 2004. 
CASTELLAN, G. Fundamentos de físico-química. Rio de Janeiro: LTC, 2001.

CHANG, R. e GOLDSBY, K. A. Química. $11^{\mathrm{a}}$ ed. Porto Alegre: AMGH Editora Ltda, 2011.

COHEN, L.; MANION, L. e MORRISON, K. Research methods in education. $6^{\mathrm{a}}$ ed. Abingdon: Routledge, 2007.

DU PLESSIS, A. Student-teachers' pedagogical beliefs: learner-centred or teacher-centred when using ICT in the science classroom? Journal of Baltic Science Education, v. 15, n. 2, p. 140-158, 2016.

DURLAK, J. A. How to select, calculate, and interpret effect sizes. Journal of Pediatric Psychology, v. 34, n. 9, p. 917-928, 2009.

DUVAL, R. Registros de representação semiótica e funcionamento cognitivo do pensamento. Trad. M. T. Moretti. Revista Eletrônica de Educação Matemática, v. 7, n. 2, p. 266-297, 2012.

EDWARDS, N. Multimodality in science education as productive pedagogy in a PGCE programme. Perspectives in Education, v. 33, n. 3, p. 159-175, 2015.

EKICI, F. T. e PEKMEZCI, S. Using ICT-supported narratives in teaching science and their effects on middle school students. Turkish Online Journal of Educational Technology, v. 14, n. 4, p. 173-186, 2015.

ENGEL, T. e REID, P. Química física. Madrid: Pearson Educación, 2006.

FIELD, A. P. Discovering statistics using IBM SPSS statistics: and sex and drugs and rock 'n' roll. $3^{\mathrm{a}}$ ed. Los Angeles: SAGE, 2009.

FOZDAR, B. I. Open and distance learning (ODL): a strategy of development through its potential role in improving science \& technology knowledge. International Journal of Emerging Technologies in Learning, v. 10, n. 2, p. 9-16, 2015.

GIORDAN, M. Análise e reflexões sobre os artigos de educação em química e multimídia publicados entre 2005 e 2014. Química Nova na Escola, v. 37, n. 2, p. 154-160, 2015.

GROFF, J. e MOUZA, C. A framework for addressing challenges to classroom technology use. Association for the Advancement of Computing in Education Journal, v. 16, n. 1, p. 21-46, 2008.

HERNÁNDEZ SAMPIERI, R.; FERNÁNDEZ COLLADO, C. e BAPTISTA LUCIO, P. Metodología de la investigación. $5^{\mathrm{a}}$ ed. Ciudad de México: McGraw-Hill Interamericana, 2010.

HILL, M. e SHARMA, M. D. Students' representational fluency at university: a cross-sectional measure of how multiple representations are used by physics students using the representational fluency survey. Eurasia Journal of Mathematics, Science and Technology Education, v. 11, n. 6, p. 1633-1655, 2015.

HinostrozA, J. E.; IBIETA, A. I.; ClARO, M. e LABBÉ, C. Characterisation of teachers' use of computers and internet inside and outside the classroom: the need to focus on the quality. Education and Information Technologies, v. 21, n. 6, p. 1595-1610, 2016.

KOEHLER, M. J. e MISHRA, P. What is technological pedagogical content knowledge? Contemporary Issues in Technology and Teacher Education, v. 9, n. 1, p. 60-70, 2009.

KOZMA, R. e RUSSELL, J. Students becoming chemists: developing representational competence. In: GILBERT, J. K. (Ed.). Visualization in science education. Dordrecht: Springer, 2005. p. 121-145.
KURT, S. Examining teachers' use of computer-based technologies: a case study. Education and Information Technologies, v. 18, p. 557$570,2013$.

LABURÚ, C. E.; BARROS, M. A. e SILVA, O. H. M. Multimodos e múltiplas representações, aprendizagem significativa e subjetividade: três referenciais conciliáveis da educação científica. Ciência \& Educação, v. 17, n. 2, p. 469-487, 2011.

LEVINE, I. N. Physical chemistry. $6^{\text {a }}$ ed. New York: McGraw-Hill, 2009.

LÓPEZ, V. e PINTÓ, R. Identifying secondary-school students' difficulties when reading visual representations displayed in physics simulations. International Journal of Science Education, p. 1-28, 2017.

MACCARTHY, B. L.; LEWIS, M.; VOSS, C. e NARASIMHAN, R. The same old methodologies? Perspectives on OM research in the post-lean age. International Journal of Operations and Production Management, v. 33, n. 7, p. 934-956, 2013.

MAHER, J. M.; MARKEY, J. C. e EBERT-MAY, D. The other half of the story: effect size analysis in quantitative research. CBE - Life Sciences Education, v. 12, p. 345-351, 2013.

MCDERMOTT, M. A. e HAND, B. The impact of embedding multiple modes of representation within writing tasks on high school students' chemistry understanding. Instructional Science, v. 41, n. 1, p. $217-$ 246, 2013.

MORENO, E. L. e HEIDELMANN, S. P. Recursos instrucionais inovadores para o ensino de química. Química Nova na Escola, v. 39, n. 1, p. 12-18, 2017.

MORTIMER, E. F. e SCOTT, P. Atividade discursiva nas salas de aula de ciências: uma ferramenta sociocultural para analisar e planejar o ensino. Investigações em Ensino de Ciências, v. 7, n. 3, p. 283-306, 2002.

; MASSICAME, T.; BUTY, C. e TIBERGHIEN, A. Uma metodologia de análise e comparação entre a dinâmica discursiva de salas de aulas de ciências utilizando software e sistema de categorização de dados em vídeo: parte 1, dados quantitativos. In: Atas do V Encontro Nacional de Pesquisa em Ensino de Ciências. Bauru, SP, 2005.

MORTIMER, R. G. Physical chemistry. $3^{\mathrm{a}}$ ed. Oxford: Elsevier Academic Press, 2008.

NIELSEN, J. Usability 101: introduction to usability. 2003. Disponível em http://tfa.stanford.edu/download/IntroToUsability.pdf, acessada em Novembro 2018

NOKELAINEN, P. An empirical assessment of pedagogical usability criteria for digital learning material with elementary school students. Educational Technology \& Society, v. 9, n. 2, p. 178-197, 2006.

NOVAK, J. D. e GOWIN, D. B. Aprender a aprender. Lisboa: Plátano, 1996.

ÖZMEN, H. Some student misconceptions in chemistry: a literature review of chemical bonding. Journal of Science Education and Technology, v. 13, n. 2, p. 147-159, 2004.

PAIVA, J. C. e COSTA, L. A. Exploration guides as a strategy to improve the effectiveness of educational software in chemistry. Journal of Chemical Education, v. 87, n. 6, p. 589-591, 2010. 
PETRUCCI, R. H.; HERRING, F. G.; MADURA, J. D. e BISSONNETTE, C. Química general. $10^{\mathrm{a}}$ ed. Madrid: Pearson Educación, 2011.

SCOTT, P.; MORTIMER, E. e AMETLLER, J. Pedagogical link-making: a fundamental aspect of teaching and learning scientific conceptual knowledge. Studies in Science Education, v. 47, n. 1, p. 3-36, 2011.

SILVA, A. C. T. e MORTIMER, E. F. Caracterizando estratégias enunciativas em uma sala de aula de química: aspectos teóricos e metodológicos em direção à configuração de um gênero do discurso. Investigações em Ensino de Ciências, v. 15, n. 1, p. 121-153, 2010.
STEINER, D. e MENDELOVITCH, M. "I'm the same teacher": the attitudes of science and computer literacy teachers regarding integrating ICT in instruction to advance meaningful learning. Eurasia Journal of Mathematics, Science and Technology Education, v. 13, n. 5, p. 1259-1282, 2017.

TRO, N. J. Chemistry: structure and properties. Upper Saddle River: Pearson Education, 2015.

TUCKMAN, B. Manual de investigação em educação. $4^{\mathrm{a}}$ ed.. Lisboa: Fundação Calouste Gulbenkian, 2012.

ZHAO, Y.; PUGH, K.; SHELDON, S. e BYERS, J. L. Conditions for classroom technology innovations. Teachers College Record, v. 104, n. 3, p. 482-515, 2002. 